\title{
Adult Learning Deficits after Neonatal Exposure to D-Methamphetamine: Selective Effects on Spatial Navigation and Memory
}

\author{
Charles V. Vorhees, Sandra L. Inman-Wood, LaRonda L. Morford, Harry W. Broening, Masao Fukumura, and \\ Mary S. Moran \\ Division of Developmental Biology, Children's Hospital Research Foundation and Department of Pediatrics, University of \\ Cincinnati, Cincinnati, Ohio 45229-3039
}

The effects of neonatal D-methamphetamine (MA) treatment on cued and spatial learning and memory were investigated. MA was administered to neonatal rats on postnatal days 11-20. All groups received four subcutaneous injections per day. Group MA40-4 received $40 \mathrm{mg} \cdot \mathrm{kg}^{-1} \cdot \mathrm{d}^{-1}$ of MA in four divided doses (10 mg/kg per injection). Group MA40-2 received 40 $\mathrm{mg} \cdot \mathrm{kg}^{-1} \cdot \mathrm{d}^{-1}$ of MA in two divided (20 mg/kg/injection) and saline for the other two injections per day. Controls received saline for four injections per day. As adults, both MA groups showed no differences in swimming ability in a straight swimming channel. The MA40-4 group showed no differences in cued learning, but was impaired in hidden platform learning in the Morris water maze on acquisition. They also showed reduced memory performance on probe trials. Similar trends were seen on reversal learning and reversal probe trials. Re- duced platform-size learning trials caused spatial learning impairments to re-emerge in the MA40-4 group. The MA40-2 group showed no differences in straight channel swimming, but was slower at finding the visible platform during cued learning. They were also impaired during acquisition and memory trials in the Morris hidden platform maze. They showed a similar trend on reversal learning and memory trials, but were not different during reduced platform-size learning trials. When the MA40-2 group's performance on hidden platform learning and memory trials was adjusted for cued trial performance, the spatial learning deficits remained. Deficits of spatial learning and memory are a selective effect of neonatal methamphetamine treatment irrespective of other learning and performance variables.

Key words: methamphetamine; development; spatial learning; Morris maze; rats; substituted amphetamines
In some cities methamphetamine (MA) use exceeds that of cocaine, and nationally the prevalence of MA use is comparable to that of crack cocaine (Johnston et al., 1998). Although there is evidence that prenatal cocaine is associated with neurobehavioral impairments (Richardson et al., 1996; Lester et al., 1998; Leech et al., 1999), there is no comparable information on MA. Human MA studies have focused on perinatal complications (Oro and Dixon, 1987; Little et al., 1988; Dixon and Bejar, 1989), with only one report on possible neonatal neurobehavioral disturbances (Dixon, 1989).

Earlier experimental investigations of developmental MA have found few effects, including no impairments of learning or memory (Martin, 1975; Martin et al., 1976, 1979, 1983; Sato and Fujiwara, 1986; Weissman and Caldecott-Hazard, 1993). Recently, we reported impairments induced by developmental MA treatment on spatial learning and memory (Vorhees et al., 1994a, 1998, 1999), whereas sequential learning in a multiple-T water maze was spared. However, the selectivity of the effect for distal, as opposed to proximal, cue learning is not yet known.

Spatial learning and memory in the Morris water maze (MWM) is linked to hippocampal function (Brandeis et al., 1989;

Received Oct. 8, 1999; revised March 29, 2000; accepted April 4, 2000.

This work was supported by National Institutes of Health research Grant DA06733 (C.V.V.), training Grant ES07051 (H.W.B. and S.L.I-W.), and individual National Research Service Award Grant DA05740 (H.W.B.).

Correspondence should be addressed to Dr. Charles V. Vorhees, Division of Developmental Biology, Children's Hospital Research Foundation, 3333 Burnet Avenue, Cincinnati, OH 45229-3039. E-mail: charles.vorhees@chmcc.org. Copyright (C) 2000 Society for Neuroscience $0270-6474 / 00 / 204732-08 \$ 15.00 / 0$
Morris, 1989, 1991; Morris et al., 1989, 1990a; McNamara and Skelton, 1993). Disruption of hippocampal function by lesions, gene targeting, or pharmacological inhibition of glutamatergic NMDA receptors impairs MWM spatial learning and memory while sparing cued learning (Morris et al., 1982, 1986, 1990b; Silva et al., 1992; Morris, 1993; Giese et al., 1998). Furthermore, it has recently been suggested that MWM-related stress may contribute to some of these impairments (Holscher, 1999). NMDA antagonist-induced spatial learning impairments in the MWM are reduced or eliminated by previous water maze experience (Bannerman et al., 1995; Saucler and Cain, 1995) as are those after saturation of LTP (Otnaess et al., 1999). Whether these effects are the product of stress reduction or of transfer of training, in which animals learn general task characteristics in the nonspatial MWM that facilitates later learning of the spatial version, is not clear.

The present experiment sought to test the selectivity of the developmental effects of MA in three ways. (1) We compared learning in the MWM under cued versus spatial conditions to determine selectivity. (2) We conducted cued learning first so that positive transfer of training or stress habituation effects in the cued version would reduce nonspecific group differences on the spatial version of the task. (3) We added a component to the MWM that increased the spatial demands of the task by reducing platform size. We reasoned that if the effect of MA is selective for distal cues, then cued learning using proximal cues should be unaffected. Furthermore, we reasoned that if stress or transfer of training effects contribute to MA-induced spatial learning impairments, then previous experience in the cued MWM or a 
straight swimming channel should attenuate differences on spatial learning. Finally, we reasoned that if MA-induced MWM effects are specific to spatial ability then increasing the spatial demands of the task (by reducing platform size) should affect MA-treated progeny selectively compared to controls.

\section{MATERIALS AND METHODS}

\section{Subjects}

Nulliparous Sprague Dawley CD (Charles River, Raleigh, NC) rats were obtained and bred in house. The day a sperm plug was obtained was considered embryonic day zero (E0). The day of parturition (P0) was considered E22. On the first day after birth (P1), offspring were weighed and randomly culled to eight, balancing for gender. Litters with gender imbalances $>5: 3$ or less than four progeny were excluded. Experimenters were blind to treatment group assignments. Litters were weaned on P28, and two males and two females were randomly selected and retained from each litter. These offspring were housed in same-gender pairs to P42 and individually thereafter. Offspring were weighed daily from P11-P21 and weekly thereafter.

\section{Experimental procedures}

There were three treatment groups with 15-18 litters in each group. There were two MA-treated groups because, in adult rats, MA administration in four doses, spaced every $2 \mathrm{hr}$, has been suggested to be more neurotoxic than other patterns (Sonsalla and Heikkila, 1988; Sonsalla et al., 1989, 1991; Bowyer et al., 1992, 1994). Because our previous developmental MA experiments had used two doses per day, we compared equal daily doses given as either two or four doses. Entire litters were treated with MA on P11-P20 with four subcutaneous injections spaced every $2 \mathrm{hr}$ (time $0,2,4$, and 6). This exposure period was based on previous experiments in which these days were found to be sensitive to the induction of MWM impairments compared to days P1-P10 (Vorhees et al., 1994a) or compared to prenatal exposure (Acuff-Smith et al., 1995). Both MA-treated groups received a total dose of $40 \mathrm{mg} \cdot \mathrm{kg}^{-1} \cdot \mathrm{d}^{-1}$ (MA40). Group MA40-2 received $40 \mathrm{mg} / \mathrm{kg}$ each day in two divided doses, such that they received doses of $20 \mathrm{mg} / \mathrm{kg}$ at time 0 and $6 \mathrm{hr}$ and saline at time 2 and $4 \mathrm{hr}$. The MA40-4 group received their $40 \mathrm{mg} / \mathrm{kg}$ each day in four divided doses, such that they received doses of $10 \mathrm{mg} / \mathrm{kg}$ at time $0,2,4$, and $6 \mathrm{hr}$; and saline controls received saline at time 0,2 , 4, and $6 \mathrm{hr}$. Drug treatment consisted of D-methamphetamine $\mathrm{HCl}$ (expressed as the free base; Sigma, St. Louis, MO) dissolved in saline in a dosing volume of $3 \mathrm{ml} / \mathrm{kg}$. During treatment, dams were removed from the litter and placed in a separate cage. Offspring were then removed one at a time from the nest, weighed, injected, and returned to the cage. After all offspring were treated, the dam was returned to the home cage.

\section{Behavioral methods}

Straight channel. Beginning between P50 and P56, each animal was administered four timed trials in a $15 \times 150 \mathrm{~cm}$ straight water channel with a wire ladder at one end. On each trial, the rat was placed in the channel at the opposite end from the goal (facing away from it) and allowed to find the ladder and escape. These trials were used to determine swimming proficiency and motivation to escape before maze trials. Water temperature was $22 \pm 1^{\circ} \mathrm{C}$.

Morris maze. The Morris water maze was as described by Morris (Morris, 1981, 1984). Our tank diameter was $183 \mathrm{~cm}$. The platform was camouflaged by being constructed of transparent acrylic and set against the black background of the interior of the tank. We have demonstrated that this arrangement is effective; when the platform is shifted randomly between trials, rats perform at chance levels (Vorhees and Minck, 1989). The platform was $10 \times 10 \mathrm{~cm}$ and was submerged $2 \mathrm{~cm}$ beneath the surface.

Cued learning. The platform had a visible marker mounted above it constructed of Styrofoam ( $7 \mathrm{~cm}$ diameter, $5 \mathrm{~cm}$ in height) and covered with black paper sealed in transparent cellophane. The cue was affixed to the platform on a $20 \mathrm{~cm}$ rod held in position by being fitted in a small hole in the center of the platform. The distance between the surface of the water and the bottom edge of the cue was $12 \mathrm{~cm}$. Black curtains were drawn closed around the maze to minimize extramaze cues. Rats were administered four consecutive trials per day for $6 \mathrm{~d}$. Trial time limit was $2 \mathrm{~min}$, and the intertrial interval (ITI) was $30 \mathrm{sec}$ spent on the platform plus an additional $15-20 \mathrm{sec}$ in its home cage while the platform was relocated. Start and platform positions were varied randomly on every trial.

Spatial learning-acquisition. The goal was positioned in the middle of one quadrant and start positions were randomly distributed among the four cardinal positions around the perimeter. Rats received five trials per day for six consecutive days with the curtains open. Daily sessions consisted of four acquisition trials and one probe trial (the probe trial was the last of the day). For acquisition trials, the time limit per trial was $2 \mathrm{~min}$, and the ITI was $30 \mathrm{sec}$. Animals not finding the platform within 2 min were placed on the platform at the end of the trial. Probe trials lasted $1 \mathrm{~min}$. Swim paths were recorded using a video-tracking system (San Diego Instruments, San Diego, CA). Data recorded on learning trials included latency, path length, cumulative distance from the platform (Gallagher et al., 1993), and first bearing and for probe trials were time in the target quadrant, target site crossings, cumulative distance from the platform site, and first bearing. First bearing was determined based on animal's average vector for the first $13 \mathrm{~cm}$ traveled at the start of each trial.

Spatial learning-reversal. On the next $6 \mathrm{~d}$, the platform was moved to the opposite quadrant. Rats were administered trials identical to those for acquisition (four platform trials per day and one probe trial). All procedures were identical to acquisition.

Spatial learning - reduced platform. After reversal, $6 \mathrm{~d}$ of additional trials were administered with the platform remaining in the reversal position. As before, rats were given five trials per day consisting of four platform trials and one probe trial. The only difference was that the platform was $25 \%(5 \times 5 \mathrm{~cm})$ of the size of the one used previously (i.e., $25 \mathrm{~cm}^{2}$ rather than $100 \mathrm{~cm}^{2}$ ). All other procedures were identical to acquisition and reversal. For all MWM procedures, water temperature was $\sim 22^{\circ} \mathrm{C}$.

\section{Statistical methods}

Data were analyzed using ANOVAs (general linear model). For data that had repeated measure components, split-plot ANOVAs were used with day and trial as within-factors. Gender was also treated as a within-factor to control for litter effects (Holson and Pearce, 1992). Because more than one subject of each gender was tested, data were averaged within gender. For split-plot analyses, sphericity tests for compound symmetry were used. Where significantly nonspherical, Greenhouse-Geisser adjusted $F$-ratios were used. Significant interactions were further analyzed using simple-effect ANOVAs. A posteriori group comparisons were performed by the method of Duncan. Frequency data (mortality) were analyzed by Fisher's test for uncorrelated proportions.

\section{RESULTS}

\section{Mortality and growth}

Litter characteristics are shown in Table 1. The MA40-2 group had three nonsurviving litters, and the MA40-4 group had one. This trend was not significant. Both MA groups had significantly increased mortality compare to saline controls. Although the MA40-2 group's mortality was higher than that of the MA40-4 group, this difference was not significant. A formal survival analysis could not be performed because mortality was too low, therefore, we analyzed the mean age of death by ANOVA. As can be seen in Table 1, there were no significant group differences for age at the time of death.

Analyses of body weight during treatment showed significant treatment group, $F_{(2,42)}=16.0, p<0.0001$, treatment $\times$ day, $F_{(18,378)}=47.8, p<0.0001$, treatment $\times$ interval (i.e., time of injection within a given day), $F_{(2,42)}=4.9, p<0.05$, and treatment $\times$ day $\times$ interval effects, $F_{(18,378)}=5.6, p<0.0001$. Both MA treatment groups gained weight slower than saline controls. Simple-effect ANOVAs and a posteriori group comparisons showed that both MA-treated groups weighed less than the saline group beginning on P13 and extending throughout the remainder of treatment on P20. These effects are illustrated in males in Table 2. Females showed a similar pattern.

Post-treatment body weight analyses showed no significant 


\begin{tabular}{lllll}
\hline Table 1. Litter characteristics & & & \\
$\begin{array}{l}\text { Treatment } \\
\text { group }^{a}\end{array}$ & $\begin{array}{l}\text { Number of litters } \\
\text { treated }\end{array}$ & $\begin{array}{l}\text { Number of litters } \\
\text { with }<4 \text { survivors }\end{array}$ & $\begin{array}{l}\text { Number of litters } \\
\text { tested }\end{array}$ & $\begin{array}{l}\text { Number of offspring } \\
\text { dying/total }(\%)^{b}\end{array}$ \\
\hline Saline & 15 & 0 & 15 & $\begin{array}{l}\text { Mean age at } \\
\text { death }( \pm \text { SEM) }\end{array}$ \\
MA40-4 & 16 & 1 & 15 & $1.120(1.7)$ \\
MA40-2 & 18 & 3 & 15 & $3 / 127(15.0)^{*}$ \\
\end{tabular}

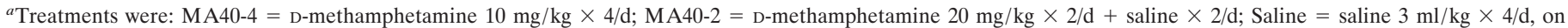
postnatal days 11 through 20 .

${ }^{b}$ There was no offspring mortality past P20.

${ }^{*} p<0.01$ compared to saline controls by Fisher's test for uncorrelated proportions.

\begin{tabular}{|c|c|c|c|c|}
\hline \multirow{2}{*}{$\begin{array}{l}\text { Age } \\
\text { (d) }\end{array}$} & \multicolumn{4}{|c|}{ Treatment group } \\
\hline & Saline & MA40-2 & MA40-4 & $F$ test \\
\hline 11 & $23.8 \pm 0.6$ & $22.8 \pm 0.7$ & $22.0 \pm 0.7$ & NS \\
\hline 15 & $31.8 \pm 0.7$ & $27.1 \pm 0.7^{* *}$ & $26.8 \pm 0.9^{* *}$ & $p<0.001$ \\
\hline 19 & $41.1 \pm 0.9$ & $32.7 \pm 0.8^{* *}$ & $31.9 \pm 1.1^{* *}$ & $p<0.001$ \\
\hline 28 & $88.1 \pm 1.9$ & $70.3 \pm 2.6^{* *}$ & $71.6 \pm 3.2 * *$ & $p<0.001$ \\
\hline 35 & $140.1 \pm 2.8$ & $120.4 \pm 4.0^{*}$ & $125.1 \pm 6.8^{*}$ & $p<0.05$ \\
\hline 42 & $199.7 \pm 4.0$ & $180.4 \pm 5.2^{*}$ & $180.6 \pm 6.5^{*}$ & $p<0.05$ \\
\hline 49 & $257.9 \pm 4.4$ & $244.7 \pm 6.1$ & $241.7 \pm 7.8$ & NS \\
\hline 56 & $306.1 \pm 5.0$ & $296.0 \pm 7.5$ & $293.6 \pm 10.6$ & NS \\
\hline 63 & $342.6 \pm 5.0$ & $333.5 \pm 7.6$ & $326.2 \pm 9.8$ & NS \\
\hline 70 & $365.0 \pm 6.7$ & $357.9 \pm 8.5$ & $347.8 \pm 9.8$ & NS \\
\hline 77 & $387.9 \pm 6.8$ & $383.6 \pm 8.6$ & $370.3 \pm 10.9$ & NS \\
\hline 84 & $407.9 \pm 6.8$ & $404.1 \pm 8.6$ & $392.2 \pm 9.3$ & NS \\
\hline 91 & $423.4 \pm 7.4$ & $428.1 \pm 8.8$ & $404.8 \pm 11.2$ & NS \\
\hline
\end{tabular}

Body weight was analyzed in two or three phases using repeated-measure ANOVA. One ANOVA was on body weight during treatment (P11-P20) and showed significant group and group by day effects. In the two-phase analysis, the second ANOVA was on body weight from the day after the last treatment to the end of the experiment (P21-P91) and showed no significant group or group by day effects. In the three-phase analysis, the second ANOVA was on body weight from the first day after treatment to the day before behavioral testing (P21-P49) and showed a significant group effect, but no interactions. In the three phase analysis, the third ANOVA analysis was on body weight during behavioral testing (P56-P91) and showed no significant group or group by day effects. Simple-effect ANOVAs are summarized in the right-hand column.

${ }^{*} p<0.05,{ }^{*} p<0.01$ compared to saline controls by pairwise a posteriori Duncan tests.

treatment group or treatment-related interactions when analyzed from P21 to P91. However, an inspection of the data showed that there was a catch-up phase to the recovery of body weight among the MA-treated groups, therefore, the data were reanalyzed in two separate ANOVAs. The first of these was for the period from the end of treatment to the day before behavioral testing began (P21-P49). In this analysis, treatment group was significant, $F_{(2,42)}=6.4, p<0.01$. Simple effect ANOVAs on each day showed that treatment group differences were present on days P21, 28, 35, and 42, but not on P49 (Table 2). No treatmentrelated interactions were significant. A posteriori group comparisons showed that both MA groups weighed less than controls on days P21-P42, but the differences progressively diminished over successive weeks until they were no longer significant by P49. The second analysis was for body weights during behavioral testing (P56-P91). No treatment group or treatment-related interactions were found (Table 2, males). Females showed a similar pattern (data not shown).

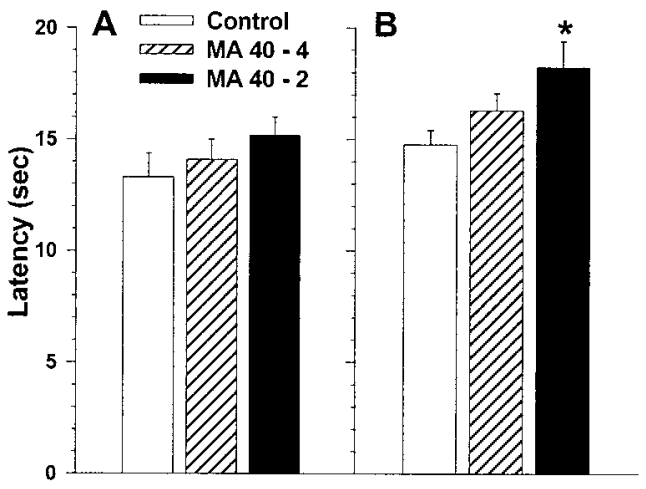

Figure 1. A, Straight channel swimming times averaged ( \pm SEM) across test trials and gender. The straight channel was $15 \times 150 \mathrm{~cm}$. $B$, Morris cued-platform maze latencies (means \pm SEM) averaged across trials (4 trials per day for $6 \mathrm{~d}$ ) and gender in MA-treated and control offspring. For these trials curtains were drawn around and over the maze, a flag was mounted above the platform, and the position of the platform was moved on every trial. Start positions also varied on every trial. ${ }^{*} p<0.05$ compared to controls by a posteriori comparisons.

\section{Straight channel swimming}

A treatment group by sex by trial split-plot ANOVA on latencies in straight swimming trials showed no significant treatment group or treatment-related interactions (Fig. 1A).

\section{Morris maze \\ Cued learning}

A treatment group by sex by day by trial split-plot ANOVA on latencies to find the platform on cued trials showed a significant treatment group main effect, $F_{(2,42)}=3.8, p<0.05$. No significant treatment-related interactions were found. A posteriori group comparisons showed that the MA40-2 group had significantly longer latencies to reach the platform than the saline group (Fig. $1 B)$. The MA40-4 group was not different from saline controls.

\section{Spatial learning and memory-acquisition}

Treatment group by sex by day by trial split-plot ANOVAs on latency, path length, and cumulative distance from the platform all showed significant treatment group main effects, latency, $F_{(2,42)}=13.3, p<0.0001$, path length, $F_{(2,42)}=8.4, p<0.001$, and cumulative distance, $F_{(2,42)}=8.1, p<0.01$. A posteriori comparisons all showed the same pattern, i.e., that both MA groups were impaired in reaching the hidden platform compared to saline controls (all comparisons $p<0.01$; Fig. $2 A-C$ ). In addition, latency and path length showed small but significant treatment $\times$ day $\times$ trial interactions, latency $F_{(30,630)}=1.8, p<0.05$, and path length, $F_{(30,630)}=1.6, p<0.05$.

Inspection of the learning curves revealed that on day 1, trial 1, 


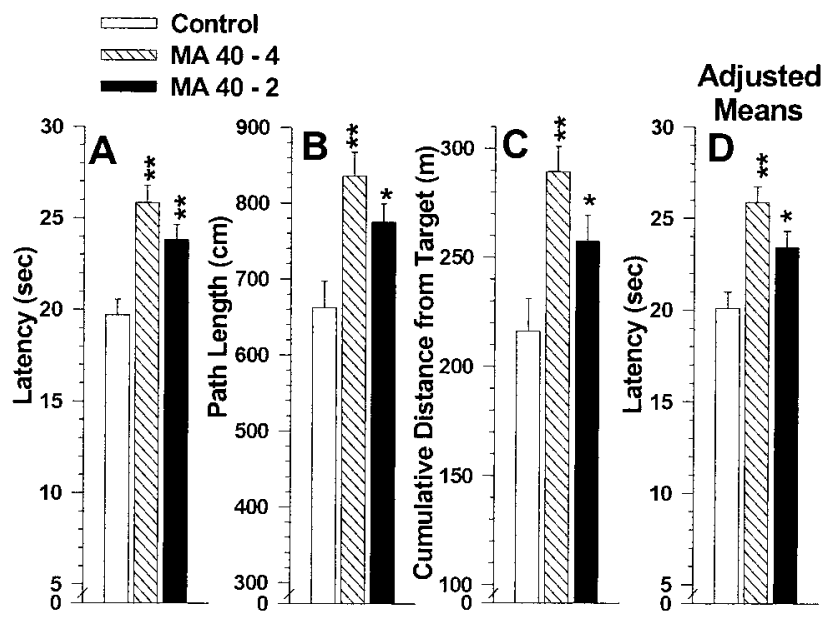

Figure 2. Morris hidden-platform maze acquisition performance in MA-treated and control offspring. For hidden platform trials, curtains were opened revealing surrounding room cues, the platform was submerged, and it was placed in a fixed position on each trial. Start positions were varied on each trial. Panels are for latency $(A)$, path length $(B)$, cumulative distance $(C)$, and latency adjusted for cued platform performance $(D)$ to find the platform (means \pm SEM) averaged across trials $(4$ trials per day for $6 \mathrm{~d}$ ) and gender. ${ }^{*} p<0.05$; ${ }^{* *} p<0.01$ compared with their respective controls by a posteriori comparisons.

all groups performed identically, i.e., they all had equivalent latencies, path lengths, and distances from the platform and showed no preference for any one quadrant over another. On successive trials, controls improved rapidly, whereas both MA groups improved by only half as much as controls. By the end of the first four trials (day 1), controls were performing significantly better than either MA-treated group. On day 2, the groups showed a similar pattern, with the same relative differences prevailing. By day 3, controls reached asymptotic performance and showed no significant further improvement through day 6 of acquisition. The MA-treated groups, by contrast, lagged behind controls on all measures on days 3-6, but continued to improve slowly, thereby narrowing the group differences caused by the ceiling effect operating in controls. Despite narrowing the gap, however, the MA-treated groups never performed as well as controls on acquisition. This was most evident on trial 1 of each day in that controls showed near optimum performance, whereas MA-treated animals showed overnight loss of accuracy and had renewed difficulty finding the platform at the start of each day.

Analyses of probe trial performance (Fig. 3) showed significant main effects of treatment group for both percentage of time in the target quadrant, $F_{(2,42)}=4.5, p<0.02$, and cumulative distance from target, $F_{(2,42)}=3.8, p<0.05$. No significant interactions between treatment group and other factors were found. A posteriori group comparisons of the main effect showed that the MA groups on both measures performed below saline controls in that they spent less time in the target quadrant (Fig. $3 A$ ) and were further away from the target site than were saline controls (Fig. $3 B)$.

Although the treatment by trial interaction for percentage of time in the target quadrant was not significant, there was a significant main effect of trial $(p<0.0001)$. This effect reflected the fact that all groups spent an increasing percentage of time in the target quadrant on probe trials across successive days. Controls spent $41 \%$ of their time in the target quadrant on the first probe trial (chance performance $=25 \%$ ) given after the first four

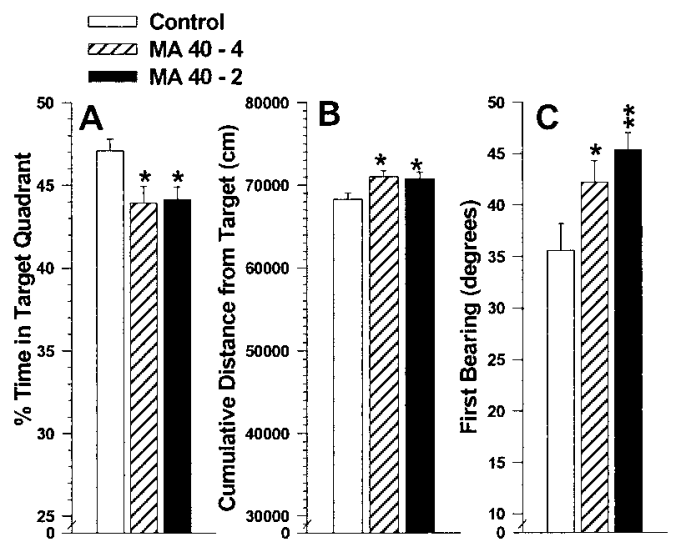

Figure 3. Morris hidden-platform maze probe trial performance (acquisition) in MA-treated and control offspring. Panels are for percent time in the target quadrant $(A)$, cumulative distance from target $(B)$, and first bearing to the target $(C)$ (means \pm SEM) averaged across probe trials and gender. ${ }^{*} p<0.05$; ${ }^{* *} p<0.01$ compared to controls by a posteriori comparisons.

learning trials on day 1 . This increased to $50 \%$ of their time in the target quadrant after their final probe trial given after the last learning trial on day 6 ( $24^{\text {th }}$ trial). By comparison, the MA40-4 group spent $34 \%$ of their time in the target quadrant on the first probe trial and $48 \%$ in the target quadrant on the last probe trial of acquisition. The MA40-2 group performed similarly to the MA40-4 group. Hence, the magnitude of the MA-induced effect on probe trials was $\sim 17 \%$ on day 1 and $4 \%$ on day 6 , reflecting the fact that MA-treated animals continued to learn after controls reached asymptotic performance (ceiling effect), thereby narrowing the difference by the end of day 6 .

An analysis of platform site crossings was not significant, although a trend was observed in the treatment group $\times$ day interaction $(p=0.07)$. This interaction showed that the MA groups had fewer platform site crossings on later probe trials than saline controls (data not shown). Probe trial performance was further analyzed for first bearing shortly after the beginning of each trial. This is the animal's initial heading after it turns away from the wall and begins to swim. The bearing is the angle of deviation between a direct lay-line to the target and the animal's heading. Analysis of first bearing showed a significant main effect of treatment group, $F_{(2,42)}=5.3, p<0.01$; no interactions with treatment group were found. $A$ posteriori group comparisons showed that both MA groups' first bearing was significantly further away from a direct line to the target than was that of the saline control group (Fig. 3C).

\section{Spatial learning and memory-reversal}

Analyses of reversal learning for latency, path length, and cumulative distance from the platform were uniform in finding no significant treatment group or treatment-related interactions (Fig. $4 A-C)$. For two of these analyses, however, an interaction trend was observed. This was the treatment $\times$ trial trend for latency $(p<0.08)$, and cumulative distance $(p<0.06)$. For both of these measures, the trend was that the MA groups, and especially the MA40-4 group, to have longer latencies and cumulative distances from the target on later trials than did saline controls. Analysis of first bearing showed a significant treatment group main effect, $F_{(2,41)}=3.6, p<0.05$ and a significant treatment group by sex by trial interaction, $F_{(6,123)}=2.8, p<0.02$. $A$ posteriori group comparisons for the main effect showed that both 


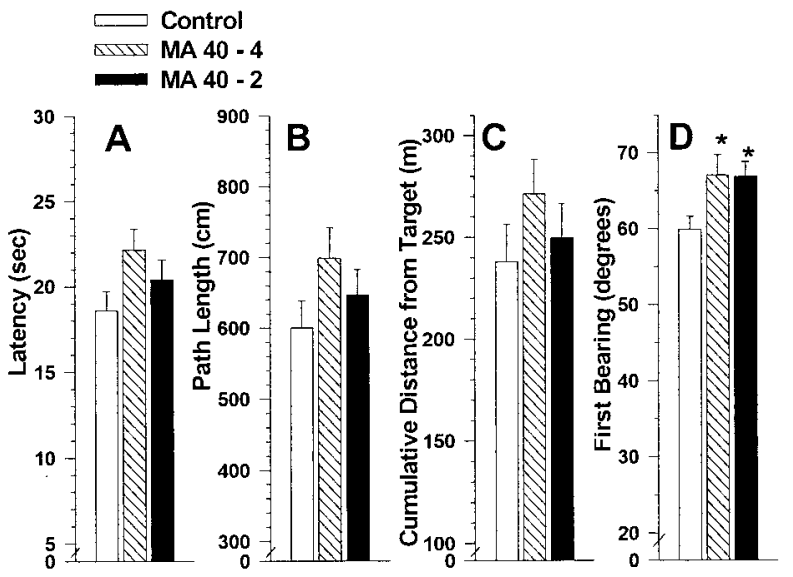

Figure 4. Morris hidden-platform maze reversal performance in MAtreated and control offspring. For reversal, the platform was moved to the opposite quadrant from that used during acquisition. Values are means \pm SEM averaged across trials and gender. Panels are latency $(A)$, path length $(B)$, cumulative distance from the target $(C)$, and first bearing to the target $(D) .{ }^{*} p<0.05$ compared to control by a posteriori group comparisons.

MA groups were more off course in their initial headings than were saline controls (Fig. 4D). Further analyses of the interaction revealed that most of the group differences were among the males. The average first bearing change among male controls from trial 1 to trial 4 (averaged across days) was $9.1^{\circ}$, whereas for the MA40-4 males it was $7.0^{\circ}$, and for the MA40-2 group it was $-3.3^{\circ}$. This indicates that on reversal the MA40-2 males had the greatest difficulty learning the new position of the platform. Females showed a similar but slightly smaller treatment effect.

On reversal probe trials, no treatment group main effects were significant. However, several interactions with treatment were obtained. Analysis of the percentage of time in the target quadrant showed significant treatment $\times \operatorname{sex}, F_{(2,41)}=3.3, p<0.05$, and treatment $\times$ day $\times$ sex effects, $F_{(10,205)}=1.9, p<0.05$; cumulative distance from the target showed a significant treatment $\times$ sex effect, $F_{(2,41)}=4.5, p<0.02$; and platform site crossings showed a significant treatment $\times$ sex $\times$ day effect, $F_{(10,205)}=1.9, p<0.05$. All of these interactions showed the same pattern, i.e., that the MA groups had greater difficulty in giving up searching where the platform used to be on acquisition and finding it in its new location (data not shown). The interactions with day and sex were the result of the male MA40-4 animals having more difficulty on days 1-3 than the MA40-2 males or the MA40-2 females or than male or female controls.

\section{Spatial learning and memory-reduced target}

Analyses of latency, path length, and cumulative distance from target showed significant treatment group main effects on all three measures: latency, $F_{(2,42)}=8.2, p<0.001$, path length, $F_{(2,42)}=7.4, p<0.01$, and cumulative distance from platform, $F_{(2,42)}=6.8, p<0.01$. There were no treatment-related interactions found. A posteriori group comparisons showed that on all three measures, the MA40-4 group had greater difficulty finding the hidden platform than controls ( $p$ values $<0.01)$. However, the MA40-2 group's performance was not significantly different from that of saline controls (Fig. $5 A-C$ ).

Analyses of probe trial performance on reduced target size trials showed no significant treatment group or treatment-related interactions on percent time in the target quadrant (Fig. 6A),

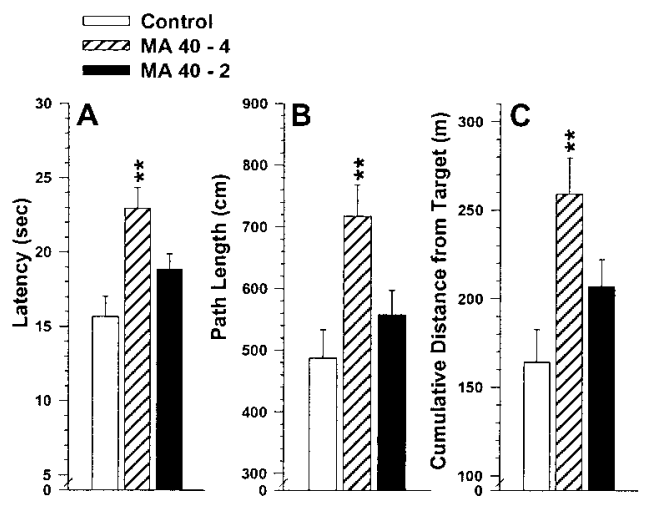

Figure 5. Morris hidden-platform maze reduced target size performance in MA-treated and control offspring. Panels are for latency $(A)$, path length $(B)$, and cumulative distance from the target $(C)$. During reduced target trials the platform was in the same position as during reversal, but the platform size was reduced from $10 \times 10 \mathrm{~cm}$ to $5 \times 5 \mathrm{~cm}$. Values are group means \pm SEM averaged across trials and gender. ${ }^{*} p<$ $0.05 ; * *<0.01$ compared to control by a posteriori group comparisons.
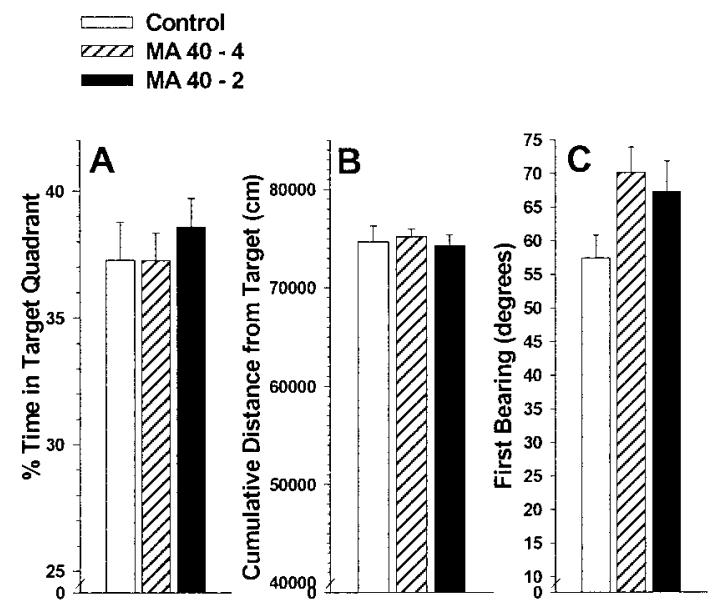

Figure 6. Morris hidden-platform reduced target size probe trial performance in MA-treated and control offspring. Values are group means \pm SEM averaged across trials and gender. Panels are percentage of time in the target quadrant $(A)$, cumulative distance from target $(B)$, and first bearing $(C)$. Note trend in first bearing in which both MA groups were further off course than controls.

cumulative distance from platform (Fig. 6B), platform site crossings (data not shown), or first bearing (Fig. 6C). A trend was present in the MA groups on first bearing performance, suggesting that the MA groups' initial heading was not as accurate as was that of the control group.

\section{Spatial learning in relation to cued learning}

As noted above, the MA40-2 group was found to have longer latencies on cued platform learning than saline controls or than the MA40-4 group. This raised the possibility that the deficits seen in the MA40-2 group on spatial navigation could be the result of performance effects that caused this group to have longer latencies on cued platform trials. To investigate this possibility, we reanalyzed the spatial learning data for acquisition, reversal, and reduced platform phases by analyses of covariance (ANCOVA), using mean latency on cued trials as the covariate. If cued trial performance accounted for the deficits in spatial learning, then the treatment effects of MA should disappear or be greatly diminished by such a covariate analysis. On the other 
hand, if the cued trial performance deficit was unrelated to performance on spatial learning, then the effects of MA on spatial measures should remain unchanged. All of the effects found on acquisition, reversal, and reduced platform remained essentially unchanged using ANCOVA. The effects of MA on spatial acquisition using adjusted means are illustrated in Figure 2D. As before, both the MA40-4 and MA40-2 groups took significantly longer to reach the platform than controls, and the effect in the MA40-2 group was only slightly altered by the covariate adjustment for cued platform performance (compare Fig. 2, $A$ and $D$ )

\section{DISCUSSION}

We hypothesized that rats exposed to MA on P11-P20 and tested as adults would show spatial navigation deficits in the Morris maze hidden platform procedure that would be selective for spatial learning and memory compared to cued learning. This prediction was confirmed in that the MA40-4 group showed no impairment in cued learning but was impaired on acquisition, reversal, and reduced target in spatial learning. This group also showed impaired memory on probe trials conducted during acquisition and reversal, however, not during reduced target trials. The MA40-2 group, on the other hand, showed a deficit in cued and spatial learning. However, when the latter was analyzed with cued performance as a covariate on spatial MWM performance, the cued MWM differences did not account for the MA-induced spatial learning difference. This indicates that the effect of the MA40-2 treatment regimen on spatial learning is not caused by the proximal cue learning difference seen in this group.

We further hypothesized that if MA-induced spatial learning impairment were the result of stress or transfer of training effects, then previous testing and handling should eliminate or reduce these differences. However, the spatial learning and memory impairments seen here were similar to those we have reported previously without previous cued learning trials in the MWM (Vorhees et al., 1998). They were also similar to those seen (Vorhees et al., 1999) with previous experience in a multiple-T swimming maze with very different task requirements (Vorhees et al., 1994a). In addition, both MA-treated and saline-treated groups in the present study were extensively handled during the neonatal period (weighing and injection four times per day). Early handling is known to reduce later responses to a stressful situation (Meaney et al., 1988). This fact, when taken together with the straight channel and cued MWM experience, indicates that it is unlikely that the effects of developmental MA treatment are accounted for by stress-related changes in physiological responses or effects derived from positive transfer of training.

Reversal trials, in which the hidden platform was moved to the opposite quadrant, revealed no significant group differences on learning trials except on first bearing. It may be that transfer of training was beneficial in this context, allowing all groups to learn the new location easier than they did during acquisition. Regardless of the basis for this change, the latency and path length measures indicate that reversal was not as difficult for the animals to learn as was acquisition, and this may explain why these measures showed no group differences on reversal. Nonetheless, the first bearing findings revealed that the MA groups did not know the location of the platform as well as controls because their bearing was significantly further from the lay-line to the target site than was that of controls. This effect was not as large as that seen on first bearing during acquisition, again suggesting that reversal was not as difficult as acquisition. It is also noteworthy that inspection of reversal first bearing patterns showed that the animals never fully corrected their first bearing to the new platform location, but rather started each trial heading for the position of the platform on acquisition and then abruptly changed course to the new position of the platform. Despite the apparent inefficiency of this pattern, it persisted through both the reversal and reducedplatform trials, and it did not prevent animals from improving their performance on each of these successive phases of testing.

We also predicted that increasing the spatial demands of the MWM would differentially affect the MA-treated progeny. This prediction was supported in that the MA40-4 group showed impaired learning when the platform size was reduced, however, the MA40-2 group did not. This suggests that the MA40-4 treatment regimen is more deleterious than the MA40-2 regimen. However, because this difference was only revealed under the more demanding requirements of locating a platform only $25 \%$ of the size of the original goal, this difference between the two dosing schedules appears to be subtle.

The MA40-4 group failed to show a significant impairment on the reduced platform probe trials. The lack of probe trial differences could be attributable to a lack of effect of MA on memory, but this seems unlikely given the probe trial deficits seen in this group on acquisition and to a lesser extent on reversal. It seems more likely that this was the result of the fact that the platform was in the same position on reduced platform trials as on reversal trials. Because of this sequence, the animals had 10 consecutive days of learning to the same quadrant position. Reduced target learning would be expected to affect platform localization, i.e., finding the platform on learning trials, but would not be expected to affect the less precise measure of time in the target quadrant given that the animals had already learned its general location from the preceding reversal trials. This is what was seen, demonstrating that with extensive training to the same target position, probe trials become less revealing of memory impairments.

It is unlikely that the effects observed in MA-treated animals can be accounted for in terms of undernutrition. First, the undernutrition caused by early MA treatment is transient and shows complete recovery. There are no residual weight reductions in the MA progeny by the time of testing. Second, MWM has been evaluated extensively in rats given severe protein-calorie malnutrition during the neonatal period (Goodlett et al., 1986; Campbell and Bedi, 1989; Bedi, 1992; Levitsky and Strupp, 1995; Strupp and Levitsky, 1995). Treatments causing more prolonged and more severe weight changes than those induced herein have consistently shown that postnatal undernutrition (and growth retardation) have no effect on spatial learning and memory in the MWM. Third, the effects of MA treatment were selective for spatial learning and memory without affecting a variety of other behaviors (Vorhees et al., 1994a,b). Such selectivity is in contrast to the kind of generalized impairment caused by malnutrition. In addition, the impairment in the MA40-4 group was more severe than that seen in the MA40-2 group, even though both groups received the same number of daily injections, had the same total daily MA dose, and showed the same body weight changes during treatment. This demonstrates that the cognitive effects of MA are dissociated from nonspecific nutritional effects. Overall, the data in this experiment reveal that differences in learning and memory are both specific to early MA treatment and selective for impairing spatial navigation.

Offspring were separated from their dams four times per day on days P11-P20, and this produces some disruption of maternalpup interactions. Whereas the exact effect of this on later spatial learning is not known, the preponderance of existing evidence 
suggests that this manipulation is most similar to that seen after early handling. Early handling, which also involves maternal-pup disruption, has been shown to improve later spatial learning and memory in the Morris maze (Meaney et al., 1988; Holscher, 1999). Although the effect of early handling and injecting was not measured in this experiment per se, all groups were matched on this variable, therefore, this factor would not be expected to contribute to group differences. All groups were individually housed for $8 \mathrm{~d}$ before to the start of testing, and individual housing has been shown to impair Morris maze performance (Wade and Maier, 1986). However, housing conditions were identical for all groups, therefore, housing would not be expected to affect the present findings unless housing were hypothesized to differentially affect MA-treated, but not control, animals. Moreover, the pretraining experience of the animals in straight channel and the cued platform maze trials would be expected to diminish group differences, yet MA-induced spatial learning and memory impairments not only remained evident, they were as large as in other experiments without these previous experiences.

Recently, it has been shown that pretraining eliminates Morris maze learning and memory impairments induced by saturation of hippocampal LTP (Otnaess et al., 1999) or by administration of NMDA antagonists that block LTP (Bannerman et al., 1995; Saucler and Cain, 1995). For example, acute treatment with the NMDA antagonist AP-5 in water maze-naïve rats results in significant $(20-33 \%)$ deficits on probe trial performance (probe trials 2 and 3 , respectively). However, this same treatment to water maze pretrained rats results in no significant deficits in spatial memory (10 and $13 \%$ differences on probe trials 2 and 3 , respectively) (Bannerman et al., 1995). Rats treated neonatally with MA showed probe trial deficits of up to $17 \%$ after pretraining in a similar maze. Hence, the developmental MA-induced memory deficits are larger than those induced by acute AP-5 treatment after water maze pretraining or after LTP saturation by high-frequency stimulation (Otnaess et al., 1999).

The spatial learning effects caused by developmental MA treatment (postnatal days 11-20) have now been shown to occur in three different strains of rats, in both males and females, in Morris mazes of different dimensions and differing procedures, with and without previous experience in other tasks, with previous experience in related and unrelated tasks, and in the absence of impairments in swimming ability. This convergence suggests that the developmental effects of methamphetamine treatment on spatial learning are reliable. This may be a cause of concern for humans exposed to this drug during stages of early brain development.

\section{REFERENCES}

Acuff-Smith KD, Schilling MA, Fisher JE, Vorhees CV (1995) Stagespecific effects of prenatal D-methamphetamine exposure on behavioral and eye development in rats. Neurotoxicol Teratol 18:199-215.

Bannerman DM, Good MA, Butcher SP, Ramsay M, Morris RGM (1995) Distinct components of spatial learning revealed by prior training and NMDA receptor blockade. Nature 378:182-186.

Bedi KS (1992) Spatial learning ability of rats undernourished during early postnatal life. Physiol Behav 51:1001-1007.

Bowyer JF, Tank AW, Newport GD, Slikker Jr W, Ali SF, Holson RR (1992) The influence of environmental temperature on the transient effects of methamphetamine on dopamine levels and dopamine release in rat striatum. J Pharmacol Exp Ther 260:817-824.

Bowyer JF, Davies DL, Schmued L, Broening HW, Newport GD, Slikker Jr W, Holson RR (1994) Further studies of the role of hyperthermia in methamphetamine neurotoxicity. J Pharmacol Exp Ther 268:1571-1580.

Brandeis R, Brandys Y, Yehuda S (1989) The use of the Morris water maze in the study of memory and learning. Int J Neurosci 48:29-69.
Campbell LF, Bedi KS (1989) The effects of undernutrition during early life on spatial learning. Physiol Behav 45:883-890.

Dixon SD (1989) Effects of transplacental exposure to cocaine and methamphetamine on the neonate. West J Med 150:436-442.

Dixon SD, Bejar R (1989) Echoencephalographic findings in neonates associated with maternal cocaine and methamphetamine use: incidence and clinical correlates. J Pediatr 115:770-778.

Gallagher M, Burwell R, Burchinal M (1993) Severity of spatial learning impairment in aging: development of a learning index for performance in the Morris water maze. Behav Neurosci 107:618-626.

Giese KP, Fedorov NB, Filipkowski RK, Silva AJ (1998) Autophosphorylation at $\mathrm{Thr}^{286}$ of the $\alpha$ calcium-calmodulin kinase II in LTP and learning. Science 279:870-873.

Goodlett CR, Valentineo ML, Morgane PJ, Resnick O (1986) Spatial cue utilization in chronically malnourished rats: task specific learning deficits. Dev Psychobiol 19:1-15.

Holscher C (1999) Stress impairs performance in spatial watermaze learning tasks. Behav Brain Res 100:225-235.

Holson RR, Pearce B (1992) Principles and pitfalls in the analysis of prenatal treatment effects in multiparous species. Neurotoxicol Teratol 14:221-228.

Johnston LD, O'Malley PM, Bachman JG (1998) National survey results on drug use. In: The monitoring the future study, 1975-1997, pp 1- 433. Rockville, MD: National Institute on Drug Abuse.

Leech SL, Richardson GA, Goldschmidt L, Day NL (1999) Prenatal substance exposure: Effects on attention and impulsivity of 6 year olds. Neurotoxicol Teratol 21:109-118.

Lester BM, LaGasse LL, Seifer R (1998) Cocaine exposure and children: the meaning of subtle effects. Science 282:633-634.

Levitsky DA, Strupp BJ (1995) Malnutrition and the brain: changing concepts and changing concerns. J Nutr 125:2212S-2220S

Little BB, Snell LM, Gilstrap LC (1988) Methamphetamine abuse during pregnancy: outcome and fetal effects. Obstet Gynecol 72:541-544.

Martin JC (1975) Effects on offspring of chronic maternal methamphetamine exposure. Dev Psychobiol 8:397-404.

Martin JC, Martin DC, Radow B, Sigman G (1976) Growth, development and activity in rat offspring following maternal drug exposure. Exp Aging Res 2:235-251.

Martin JC, Martin DC, Radow B, Day HE (1979) Life span and pathology in offspring following nicotine and methamphetamine exposure. Exp Aging Res 5:509-522.

Martin JC, Martin DC, Sigman G, Day-Pfeiffer H (1983) Saccharin preferences in food deprived aging rats are altered as a function of perinatal drug exposure. Physiol Behav 30:853-858.

McNamara RK, Skelton RW (1993) The neuropharmacological and neurochemical basis of place learning in the Morris water maze. Brain Res Rev 18:33-49.

Meaney MJ, Aitken DH, van Berkel C, Bhatnager S, Sapolsky RM (1988) Effect of neonatal handling on age-related impairments associated with the hippocampus. Science 239:766-768.

Morris R (1984) Developments of a water-maze procedure for studying spatial learning in the rat. J Neurosci Methods 11:47-60.

Morris RGM (1981) Spatial localization does not require the presence of local cues. Learn Motiv 12:239-260.

Morris RGM (1989) Synaptic plasticity and learning: selective impairment of learning in rats and blockade of long-term potentiation in vivo by the $N$-methyl-D-aspartate receptor antagonist AP5. J Neurosci 9:3040-3057.

Morris RGM (1991) Distinctive computations and relevant associative processes: Hippocampal role in processing, retrieval, but not storage of allocentric spatial memory. Hippocampus 1:287-290.

Morris RGM (1993) An attempt to dissociate "spatial-mapping" and "working-memory" theories of hippocampal function. In: Neurobiology of the hippocampus (Seifert W, ed), pp 405-432. New York: Academic.

Morris RGM, Garrud P, Rawlins JNP, O'Keefe J (1982) Place navigation impaired in rats with hippocampal lesions. Nature 297:681-683.

Morris RGM, Hagan JJ, Rawlins JNP (1986) Allocentric spatial learning by hippocampectomized rats: a further test of the "spatial mapping" and "working memory" theories of hippocampal function. Q J Exp Psychol 38B:365-395.

Morris RGM, Halliwell RF, Bowery N (1989) Synaptic plasticity and learning II: do different kinds of plasticity underlie different kinds of learning? Neuropsychologia 27:41-59.

Morris RGM, Davis S, Butcher SP (1990a) Hippocampal synaptic plasticity and NMDA receptors: a role in information storage? Philos Trans R Soc Lond B Biol Sci 329:187-204. 
Morris RGM, Schenk F, Tweedie F, Jarrard LE (1990b) Ibotenate lesions of hippocampus and/or subiculum: dissociating components of allocentral spatial learning. Eur J Neurosci 2:1016-1028.

Oro AS, Dixon SD (1987) Perinatal cocaine and methamphetamine exposure: maternal and neonatal correlates. J Pediatr 111:571-578.

Otnaess MK, Brun VH, Moser M-B, Moser EI (1999) Pretraining prevents spatial learning impairment after saturation of hippocampal longterm potentiation. J Neurosci 19(RC49):1-5.

Richardson GA, Conroy ML, Day NL (1996) Prenatal cocaine exposure: effects on the development of school-age children. Neurotoxicol Teratol 18:627-634.

Sato M, Fujiwara Y (1986) Behavioral and neurochemical changes in pups prenatally exposed to methamphetamine. Brain Dev 8:390-396.

Saucler D, Cain DP (1995) Spatial learning without NMDA receptordependent long-term potentiation. Nature 378:186-189.

Silva AJ, Paylor R, Wehner JM, Tonegawa S (1992) Impaired spatial learning in $\alpha$-calcium-calmodulin kinase II mutant mice. Science 257:206-211.

Sonsalla PK, Heikkila RE (1988) Neurotoxic effects of 1-methyl-4phenyl-1,2,3,6-tetrahydropryidine (MPTP) and methamphetamine in several strains of mice. Prog Neuropsychopharmacol Biol Psychiatr 12:345-354.

Sonsalla PK, Nicklas WJ, Heikkila RE (1989) Role for excitatory amino acids in methamphetamine-induced nigrostriatal dopaminergic toxicity. Science 243:398-400.

Sonsalla PK, Riordan DE, Heikkila RE (1991) Competitive and noncompetitive antagonists at $N$-methyl-D-aspartate receptors protect against methamphetamine-induced dopaminergic damage in mice. J Pharmacol Exp Ther 256:506-512.
Strupp BJ, Levitsky DA (1995) Enduring cognitive effects of early malnutrition: a theoretical reappraisal. J Nutr 125:2221S-2232S.

Vorhees CV, Minck DR (1989) Long-term effects of prenatal phenytoin exposure on offspring behavior in rats. Neurotoxicol Teratol 11:295-305.

Vorhees CV, Ahrens KG, Acuff-Smith KD, Schilling MA, Fisher JE (1994a) Methamphetamine exposure during early postnatal development in rats: I. Acoustic startle augmentation and spatial learning deficits. Psychopharmacology 114:392-401.

Vorhees CV, Ahrens KG, Acuff-Smith KD, Schilling MA, Fisher JE (1994b) Methamphetamine exposure during early postnatal development in rats: II. Hypoactivity and altered responses to pharmacological challenge. Psychopharmacology 114:402-408.

Vorhees CV, Reed TM, Schilling MA, Fisher JE, Moran MS, Cappon GD, Nebert DW (1998) CYP2D1 polymorphism in methamphetaminetreated rats: genetic differences in neonatal mortality and a comparison of spatial learning and acoustic startle. Neurotoxicol Teratol 20:265-273.

Vorhees CV, Morford LL, Inman SL, Reed TM, Schilling MA, Cappon GD, Moran MS, Nebert DW (1999) Genetic differences in spatial learning between Dark Agouti and Sprague-Dawley strains: possible correlation with CYP2D2 polymorphism in rats treated neonatally with methamphetamine. Pharmacogenetics 9:171-181.

Wade SE, Maier SF (1986) Effects of individual housing and stressor exposure upon the acquisition of watermaze escape. Learn Motiv 17:287-310.

Weissman AD, Caldecott-Hazard S (1993) In utero methamphetamine effects: I. Behavior and monoamine uptake sites in adult offspring. Synapse 13:241-250. 